\title{
Adipositastherapie online
}

\begin{abstract}
Verhaltensinterventionen sind in der Lage, bei adipösen Patienten einen klinisch signifikanten Gewichtsverlust über 24 Monate hinweg zu erreichen und zu halten.
\end{abstract}

- In einer randomisierten, kontrollierten Studie wurden die Auswirkungen von zwei Verhaltensinterventionen zur Gewichtsabnahme bei 415 adipösen Patienten mit mindestens einem kardiovaskulären Risikofaktor untersucht.

Eine Gruppe erhielt unterstützende Beratung zur Gewichtsabnahme per Telefon, einer speziellen Internetseite sowie über E-Mails. Die zweite Gruppe erhielt zusätzlich auch noch persönliche Unterstützung zur Gewichtsabnahme. In einer Kontrollgruppe versuchten die
Patienten von sich aus, Gewicht abzunehmen.

Der durchschnittliche Body-MassIndex zu Beginn der Studie lag bei allen Teilnehmern bei $36,6 \mathrm{~kg} / \mathrm{m}^{2}$, das durchschnittliche Gewicht bei 103,8 kg. Nach 24 Monaten hatte das Gewicht in der Gruppe mit Unterstützung über Telefon Internet und E-Mail um 4,6 kg, in der Gruppe mit zusätzlicher persönlicher Beratung um 5,1 kg und in der Kontrollgruppe um $0,8 \mathrm{~kg}$ abgenommen. Der Unterschied zwischen der Kontrollgruppe und beiden Interventionsgruppen ist statistisch hochsignifikant.

\section{- L.J. Appel et al.}

(lappel@jhmi.edu): Comparative effectiveness of weight-loss interventions in clinical practice. New Engl. J. Med. 365 (2011) 21, 1959-1968

\section{Kommentar}

Bei einem Ausgangsgewicht von $103 \mathrm{~kg}$ im Lauf von zwei Jahren mit offensichtlich erheblichem Einsatz $5 \mathrm{~kg}$ zu verlieren, kann sowohl als deprimierend als auch als vielversprechend angesehen werden. Deprimierend, weil trotz des relativ großen Aufwandes sich fünf Kilo eben ziemlich bescheiden ausnehmen. Hoffnungsvoll, weil bei Fortsetzung dieser Gewichtsabnahme über die zwei Jahre hinaus möglicherweise in zehn Jahren eine klinisch relevante Gewichtsreduktion zu erzielen sein könnte. Ob die Patienten allerdings derartige Beratungen über einen so langen Zeitraum hinweg tolerieren, erscheint doch fraglich. Eine effektive Adipositastherapie erfordert tatsächlich eine tief greifende Umstellung der Ernährungs- und Lebensgewohnheiten, zu deren konsequenter und nachhaltiger Beachtung wahrscheinlich nur eine Minderheit in der Lage ist. H. S. FüESSL =

\section{Nach Appendektomie erneut Appendizitis}

\section{Die anamnestische Angabe einer Appendektomie, die typische Narbe im rechten Unterbauch oder auch die Narben nach einem lapa- roskopischen Eingriff schließen eine Appendizitis keinesfalls aus.}

— Ein 19-Jähriger mit Zustand nach laparoskopischer Appendektomie wegen perforierter Appendizitis suchte wegen seit fünf Tagen bestehenden periumbilikalen Bauchschmerzen, die sich in den rechten unteren Quadranten verlagert hatten, die Nothilfe auf. Die Ultraschalluntersuchung ergab keinen hinweisenden Befund und der Patient wurde unter der Diagnose einer Gastroenteritis stationär aufgenommen. In der Nacht entwickelte der Patient Fieber, eine Leukozytose von 2000/ $\mu$ l und Zeichen einer Peritonitis. Das Abdomen-CT zeigte freie Flüssigkeit, freie Luft und Kotsteine rechts parakolisch (Abb. 1).

Unter der Diagnose einer komplizierten Appendizitis wurde der Patient laparatomiert. Nach Mobilisierung des Coecums zeigte sich ein großer parakolischer Abszess, in dem eine nach retro-
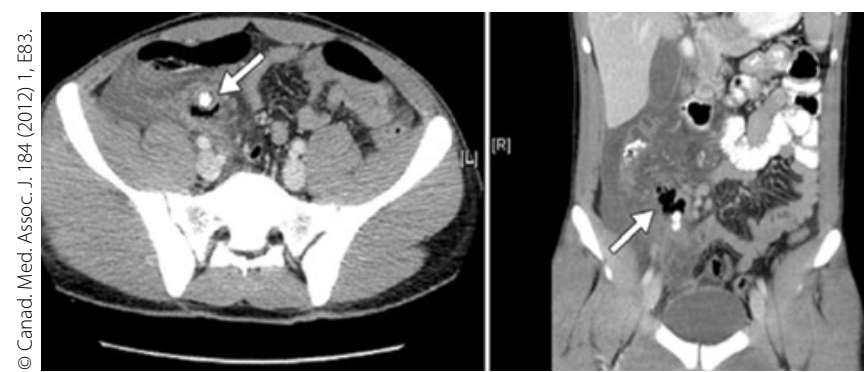

Abb.1 CT bei einem 19-Jährigen mit bekannter Appendektomie: perforierte Appendizitis mit extraluminaler Gasansammlung, freier Flüssigkeit und Fäkolithen.

zökal geschlagene, perforierte und nekrotische Appendix erkennbar war. Die Stelle der früheren Appendektomie konnte nicht identifiziert werden. Der abschließende pathologisch-anatomische Befund ergab eine eitrige Appendizitis mit Perforation. Zur Überraschung der Ärzte zeigte ein Blick in die Krankenakten des Patienten, dass diese Diagnose auch bei der ersten Appendektomie gestellt wurde. Offensichtlich lag bei dem Patienten eine Appendix-Duplikatur mit metachroner Appendizitis vor.

\section{- K. L. Martin et al.}

(Korres.: kmartin2008@meds.uwo.ca): Recurrent appendicitis after appendectomy. Canad. Med. Assoc. J. 184 (2012) 1, E83.

\section{Kommentar}

Eine retrozökal liegende Duplikatur der Appendix entsteht durch die fehlende Rückbildung einer in der sechsten Embryonalwoche vorübergehend vorhandenen Ausstülpung des Zökums. Sie soll bei etwa 0,004-0,009\% der Bevölkerung vorkommen. Der häufigste Grund für Schmerzen im rechten unteren Quadranten, Fieber und Leukozytose ist zweifelsohne die Appendizitis. Eine konventionelle Narbe im rechten Unterbauch oder die anamnestische Angabe eine Appendizitis schließen dieser Diagnose nicht aus, da immer noch die Möglichkeit einer sogenannten Stumpf-Appendizitis oder eines Rezidivs aufgrund einer Appendix-Duplikatur besteht.

H. S. FüESSL" 